\title{
ARTICLE
}

Clinical Study

\section{Apatorsen plus docetaxel versus docetaxel alone in platinum- resistant metastatic urothelial carcinoma (Borealis-2)}

\author{
Jonathan E. Rosenberg', Noah M. Hahn², Meredith M. Regan ${ }^{3}$, Lillian Werner ${ }^{3}$, Ajjai Alva ${ }^{4}$, Saby George ${ }^{5}$, Joel Picus ${ }^{6}$, Robert Alter ${ }^{7}$, \\ Arjun Balar ${ }^{8}$, Jean Hoffman-Censits ${ }^{9}$, Petros Grivas ${ }^{10}$, Richard Lauer ${ }^{11}$, Elizabeth A. Guancial ${ }^{12}$, Christopher Hoimes ${ }^{13}$, Guru Sonpavde ${ }^{14}$, \\ Constantine Albany ${ }^{15}$, Mark N. Stein ${ }^{16}$, Tim Breen $^{17}$, Cindy Jacobs ${ }^{18}$, Kirsten Anderson ${ }^{18}$, Joaquim Bellmunt ${ }^{3}$, Aly-Khan A. Lalani ${ }^{3}$, \\ Sumanta Pal ${ }^{19}$ and Toni K. Choueiri $\mathbb{D}^{3}$
}

BACKGROUND: A randomised study to assess the addition of apatorsen, an antisense oligonucleotide that inhibits Hsp27 expression, to docetaxel in patients with metastatic urothelial carcinoma ( $\mathrm{WUC}$ ) relapsed after prior platinum-based chemotherapy. METHODS: Multicentre, phase II study with 1:1 randomisation to apatorsen (three loading doses at $600 \mathrm{mg}$ intravenous followed by weekly doses) plus docetaxel $\left(75 \mathrm{mg} / \mathrm{m}^{2}\right.$ intravenous every 21 days) (A/D) or docetaxel alone. Overall survival (OS) was the primary end point with a $P$ value $<0.1$ (one-sided) being positive. Progression-free survival (PFS), objective response rate (ORR), safety, and effect of Hsp27 levels on outcomes were secondary end points.

RESULTS: Patients randomised to A/D $(n=99)$ had improved OS compared to docetaxel alone $(n=101): \mathrm{HR}: 0.80,80 \% \mathrm{Cl}$ : $0.65-0.98, P=0.0784$, median 6.4 vs 5.9 months. PFS and ORR were similar in both arms. A/D had more incidence of sepsis and urinary tract infections. Patients with baseline Hsp27 levels $<5.7 \mathrm{ng} / \mathrm{mL}$ had improved OS compared to those with levels $\geq 5.7 \mathrm{ng} /$ $\mathrm{mL}$. Patients with a decline or $\leq 20.5 \%$ increase in $\mathrm{Hsp} 27$ from baseline benefited more from A/D than those with $>20.5 \%$ increase. CONCLUSIONS: A/D met its predefined OS end point in patients with platinum-refractory mUC in this phase II trial. This trial is hypothesis generating requiring further study before informing practice.

British Journal of Cancer (2018) 118:1434-1441; https://doi.org/10.1038/s41416-018-0087-9

\section{INTRODUCTION}

Heat shock proteins $(\mathrm{Hsp})$ are a family of highly conserved proteins whose expression is induced by cell stressors such as hyperthermia, oxidative stress, cytotoxic chemotherapy, and radiation. ${ }^{1}$ Hsp27 is highly expressed in many cancers and is associated with poor prognosis. ${ }^{1,2} \mathrm{Hsp} 27$ also stabilises mutated or inappropriately activated oncoproteins that contribute to the initiation, growth, and metastasis of human cancers. ${ }^{3-7}$ While Hsp27 is expressed in low levels in normal bladder epithelium, expression is increased in bladder cancer. ${ }^{9-11}$

Apatorsen (OGX-427) is an antisense oligonucleotide (ASO) designed to bind to Hsp27 mRNA, resulting in the inhibition of the production of Hsp27 protein. ${ }^{12,13}$ Apatorsen is similar to endogenous DNA but contains second-generation ASO chemical modifications intended to optimise its pharmacological potency, pharmacokinetics, and safety profile. In vitro and in vivo evidence indicates that Hsp27 inhibition leads to inhibition of tumour growth and sensitisation to cytotoxic chemotherapy, ${ }^{14,15}$ and a trial of apatorsen as intravesical therapy for non-muscle invasive bladder cancer showed promising anticancer activity. ${ }^{16}$ Phase I studies of apatorsen as a single agent and in combination with docetaxel appeared to be well tolerated even at the highest dose of $1000 \mathrm{mg}^{1}$

We report the efficacy and safety of apatorsen in combination with docetaxel compared to docetaxel alone in patients with metastatic urothelial carcinoma previously treated with platinumbased chemotherapy. This randomised, controlled phase II trial with a primary end point of overall survival was designed to provide a strong rationale for whether to move forward with a phase III trial in this patient population.

\section{PATIENTS AND METHODS}

Study design and participants

This was a randomised, phase II, investigator-sponsored, multicentre, open-label trial conducted among academic and

\footnotetext{
${ }^{1}$ Memorial Sloan Kettering Cancer Center, New York, NY, USA; ${ }^{2}$ Sidney Kimmel Comprehensive Cancer Center, Johns Hopkins University, Baltimore, MD, USA; ${ }^{3}$ Dana-Farber Cancer

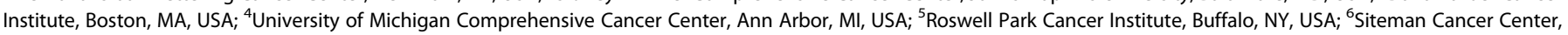

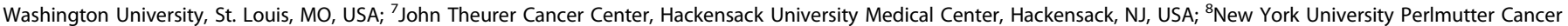

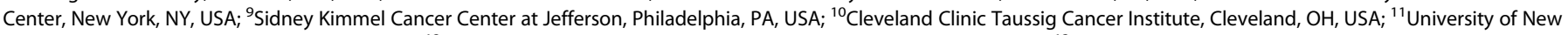

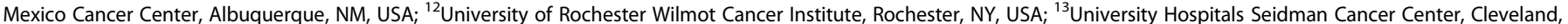

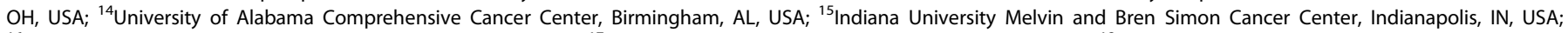

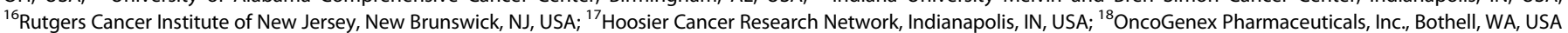
and ${ }^{19}$ City of Hope Comprehensive Cancer Center, Duarte, CA, USA

Correspondence: Toni K. Choueiri (Toni_Choueiri@DFCI.HARVARD.EDU)
}

Received: 12 October 2017 Revised: 23 March 2018 Accepted: 28 March 2018

Published online: 16 May 2018 
community sites within the Hoosier Cancer Research Network (HCRN). Patients with metastatic or locally advanced inoperable urothelial carcinoma (TNM staging T4b, N2, N3, or M1) previously treated with platinum-based chemotherapy were enrolled. To be eligible, patients 18 years or older were required to have measurable disease, an Eastern Cooperative Oncology Group (ECOG) performance status of 0 or 1 , and estimated life expectancy of 3 or more months. All patients must have received at least one prior platinum-based chemotherapy regimen with a maximum of two regimens. Patients who relapsed within 1 year of platinum-based perioperative chemotherapy were eligible. Patients whose tumours contained variant histological features were eligible if the tumour was not considered a pure histologic variant; however, patients with any amount of small cell carcinoma were not eligible. Patients were required to have adequate organ function (serum creatinine $\leq 1.5 \times$ upper limit of normal (ULN)), no worse than grade 1 peripheral neuropathy, no known brain or spinal cord metastases, no active second malignancy, no cerebrovascular accident, myocardial infarction, or pulmonary embolus within 3 months of enrollment, and no prior treatment with docetaxel.

\section{Randomisation}

Patients were randomly assigned to apatorsen plus docetaxel or docetaxel alone in a one-to-one ratio using permuted blocks within strata. Randomisation was generated using a clinical trial management system software (OnCore) and patients were stratified based on 0 vs $1-3$ adverse Bellmunt prognostic factors (liver metastases, haemoglobin $<10 \mathrm{~g} / \mathrm{dL}$, ECOG performance status 1$)$ and time from prior systemic chemotherapy ( $<3$ months vs $\geq 3$ months). ${ }^{18,19}$
Procedures

For patients assigned apatorsen plus docetaxel, apatorsen $600 \mathrm{mg}$ was administered intravenously in three separate loading doses separated by at least one non-treatment day over a 9-day period. Patients were administered an antihistamine or an $\mathrm{H} 2$ antagonist prior to each of the three loading doses. Following the loading doses, patients received docetaxel $75 \mathrm{mg} / \mathrm{m}^{2}$ in 21-day cycles and apatorsen $600 \mathrm{mg}$ weekly until disease progression, unacceptable toxicity, or a maximum of 10 cycles of docetaxel. Patients who completed 10 cycles or stopped docetaxel for toxicity continued maintenance apatorsen until disease progression or unacceptable toxicity related to apatorsen. For patients assigned docetaxel alone, docetaxel was administered at a dose of $75 \mathrm{mg} / \mathrm{m}^{2}$ every 21 days until disease progression, unacceptable toxicity, or a maximum of 10 cycles of docetaxel. Dose reductions for docetaxel (from $75 \mathrm{mg} / \mathrm{m}^{2}$ to $60 \mathrm{mg} / \mathrm{m}^{2}$ to $45 \mathrm{mg} / \mathrm{m}^{2}$ ) were required for haematologic toxicity, peripheral sensory neuropathy, or mucositis. Dose reductions for apatorsen (from $600 \mathrm{mg}$ to $500 \mathrm{mg}$ to $400 \mathrm{mg}$ ) were required for renal toxicity, and dose reductions for both agents were required for hepatotoxicity.

\section{Study end points}

The primary end point was overall survival (OS), defined from randomisation until death due to any cause, or censored on date last known alive. Secondary efficacy end points were progressionfree survival (PFS), defined from randomisation to objective disease progression or death from any cause, whichever occurred first, or censored at date of last disease evaluation without progression; objective response rate (ORR; complete or partial response as best overall response) and duration of response, which were evaluated by RECIST criteria version 1.1. Radiographic

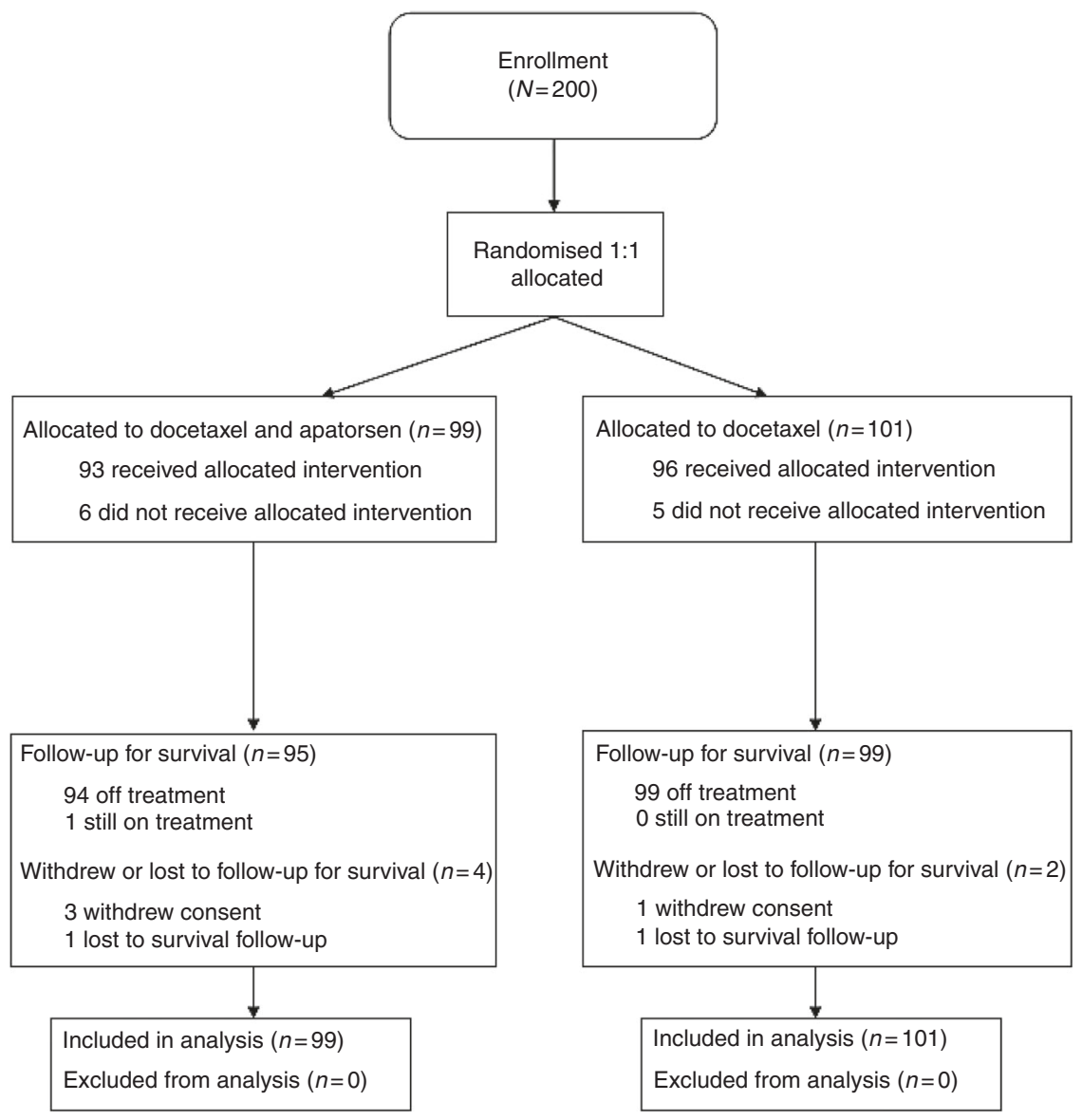

Fig. 1 Trial CONSORT flow diagram 
Table 1. Baseline characteristics

\begin{tabular}{|c|c|c|}
\hline \multirow[b]{2}{*}{ Characteristic, $n$ (\%) } & \multicolumn{2}{|c|}{ Treatment assignment } \\
\hline & $\begin{array}{l}\text { Apatorsen and } \\
\text { docetaxel }(n=99)\end{array}$ & $\begin{array}{l}\text { Docetaxel } \\
(n=101)\end{array}$ \\
\hline $\begin{array}{l}\text { Age, median years } \\
\text { (range) }\end{array}$ & $68(43-90)$ & $67(35-92)$ \\
\hline \multicolumn{3}{|l|}{ Sex } \\
\hline Male & $74(74.7 \%)$ & 75 (74.3\%) \\
\hline Female & $25(25.3 \%)$ & $26(25.6 \%)$ \\
\hline \multicolumn{3}{|l|}{ Race } \\
\hline Caucasian & $89(89.9 \%)$ & $92(91.1 \%)$ \\
\hline African American & $3(3 \%)$ & $4(4 \%)$ \\
\hline Asian & $5(5.1 \%)$ & $3(3 \%)$ \\
\hline Unknown & $2(2 \%)$ & $2(2 \%)$ \\
\hline \multicolumn{3}{|c|}{ ECOG performance status ${ }^{a}$} \\
\hline 0 & $43(43.4 \%)$ & $41(40.6 \%)$ \\
\hline 1 & $56(56.6 \%)$ & $59(58.4 \%)$ \\
\hline \multicolumn{3}{|c|}{ Urothelial carcinoma (at study entry) } \\
\hline Metastatic & $85(85.9 \%)$ & $87(86.1 \%)$ \\
\hline Locally advanced & $6(6.1 \%)$ & $10(9.9 \%)$ \\
\hline Unknown & $8(8.1 \%)$ & $4(4 \%)$ \\
\hline \multicolumn{3}{|l|}{ Primary surgery } \\
\hline Yes & $40(40.4 \%)$ & $36(35.6 \%)$ \\
\hline \multicolumn{3}{|l|}{ Prior cisplatin use } \\
\hline Yes & $70(70.7 \%)$ & $72(71.3 \%)$ \\
\hline \multicolumn{3}{|l|}{ Prior carboplatin use } \\
\hline Yes & $38(38.4 \%)$ & $41(40.6 \%)$ \\
\hline \multicolumn{3}{|l|}{ Primary disease site(s) } \\
\hline Bladder & $64(64.6 \%)$ & $72(71.3 \%)$ \\
\hline Renal pelvis & $27(27.3 \%)$ & $13(12.9 \%)$ \\
\hline Ureter & $13(13.1 \%)$ & $14(13.9 \%)$ \\
\hline Urethra & 7 (7.1\%) & 9 (8.9\%) \\
\hline \multicolumn{3}{|l|}{ Metastatic sites } \\
\hline Liver & $28(28.3 \%)$ & $25(24.8 \%)$ \\
\hline Lung & $34(34.3 \%)$ & $35(34.7 \%)$ \\
\hline Bone & 19 (19.2\%) & $21(20.8 \%)$ \\
\hline Lymph nodes & $56(56.6 \%)$ & $52(51.5 \%)$ \\
\hline \multicolumn{3}{|c|}{ Bellmunt prognostic factors ${ }^{b}$} \\
\hline 0 & $27(27.3 \%)$ & $32(31.7 \%)$ \\
\hline 1 & $42(42.4 \%)$ & $35(34.7 \%)$ \\
\hline 2 & $23(23.2 \%)$ & $26(25.7 \%)$ \\
\hline 3 & 7 (7.1\%) & 7 (6.9\%) \\
\hline
\end{tabular}

Prior paclitaxel use was balanced between both arms $(n=10 \mathrm{arm} \mathrm{A}$ and $n$ $=12 \mathrm{arm} \mathrm{B}$ ). ECOG Eastern Cooperative Oncology Group. ${ }^{\mathrm{a}}$ One patient in the docetaxel arm had ECOG performance status of $2 .{ }^{b}$ One patient in the docetaxel arm had unknown Bellmunt prognostic factors

evaluations were performed at baseline with cross-sectional imaging and repeated every 6 weeks until disease progression. Patients with bone metastases on baseline bone scan were required to have imaging every 6 weeks for the first 4 cycles and then every 12 weeks thereafter until disease progression. If any new clinical signs or symptoms of disease progression developed, imaging was repeated as clinically indicated. Safety was reported according to National Cancer Institute $(\mathrm{NCl})$ Common Terminology Criteria for Adverse Events (CTCAE version 4.0) and assessed from initiation of study treatment until 30 days after last study therapy. Exploratory objectives included assessing the associations of baseline and post-treatment serum Hsp27 levels with survival outcomes. Hsp27 levels were analysed by a central laboratory using enzyme-linked immunosorbent assay analysis, as has been previously described. ${ }^{20}$

Statistical analysis

This phase II study was designed to have $90 \%$ power with onesided 0.10 significance level to detect a $33 \%$ reduction in the OS hazard rate with docetaxel and apatorsen compared with docetaxel alone [hazard ratio (docetaxel and apatorsen/docetaxel) $=0.667]$, assuming an exponential distribution of OS, and median OS of 6 months on docetaxel alone. ${ }^{21}$ The specified phase II error levels were considered to provide adequate precision of the hazard ratios (HR) in order to inform the design of a subsequent phase III trial. The randomised, controlled design specified one interim analysis for futility after $\sim 81$ deaths and final analysis after 162 deaths. The cutoff date for final analysis was 10 October 2016.

Patient and clinical characteristics were summarised as numbers and percentage for categorical variables and median with range for continuous variables. OS and PFS were compared between the two treatment assignments using a stratified log-rank test with a one-sided $a=0.10$. The Kaplan-Meier (KM) method was used to estimate OS and PFS distributions by treatment arm. Stratified Cox proportional hazards (PH) models estimated hazard ratios (HR) and $80 \%$ two-sided confidence intervals, which corresponds to one-sided $a=0.10$, in unadjusted and multivariable models. Subgroup analyses investigated treatment effects according to the stratification factors, estimating HRs and testing for treatmentby-subgroup interaction in Cox PH models.

ORR was summarised as numbers and percentage of participants by treatment assignments with two-sided $80 \% \mathrm{Cl}$ and compared using Fisher's exact tests. Median duration of response was estimated using KM method in patients who achieved partial or complete response as best overall response, defined from time objective response was first observed until disease progression or death. A planned stratified Cox $\mathrm{PH}$ model assessed the association of baseline serum Hsp27 level with OS and to test the treatmentby-Hsp27 interaction. Baseline serum Hsp27 levels were categorized at the median for assessing the associations with OS, given that no clinically meaningful cutoff point had been previously established. Among patients who were alive after cycle 2, the association of percentage change of $\mathrm{Hsp} 27$ level from baseline to end of cycle 2 with OS (re-defined from end of cycle 2 as landmark analysis) was investigated similarly.

\section{RESULTS}

Patients

Between August 2013 and September 2015, 200 patients were enrolled at 32 study sites in the United States. Ninety-nine patients were randomised to docetaxel and apatorsen and 101 patients to treatment with docetaxel alone. Overall, 194 participants had complete follow-up for survival and 6 were lost to follow-up or withdrew consent without survival follow-up. All 200 participants were included in the intention-to-treat (ITT) analysis population (Fig. 1). Baseline characteristics were well balanced as shown in Table 1. Overall, median age of participants was 67 years (interquartile range, IQR 59-74) and 149 participants (74.5\%) were male. Eighty-four (42.0\%) had ECOG performance status of 0 at screening. In terms of patient stratification, 140 (70\%) had 1-3 Bellmunt prognostic factors and $87(43.5 \%)$ had time from prior systemic chemotherapy $<3$ months.

Therapy administration

Among patients who started docetaxel, a median of two cycles were received in both groups and among those who received 


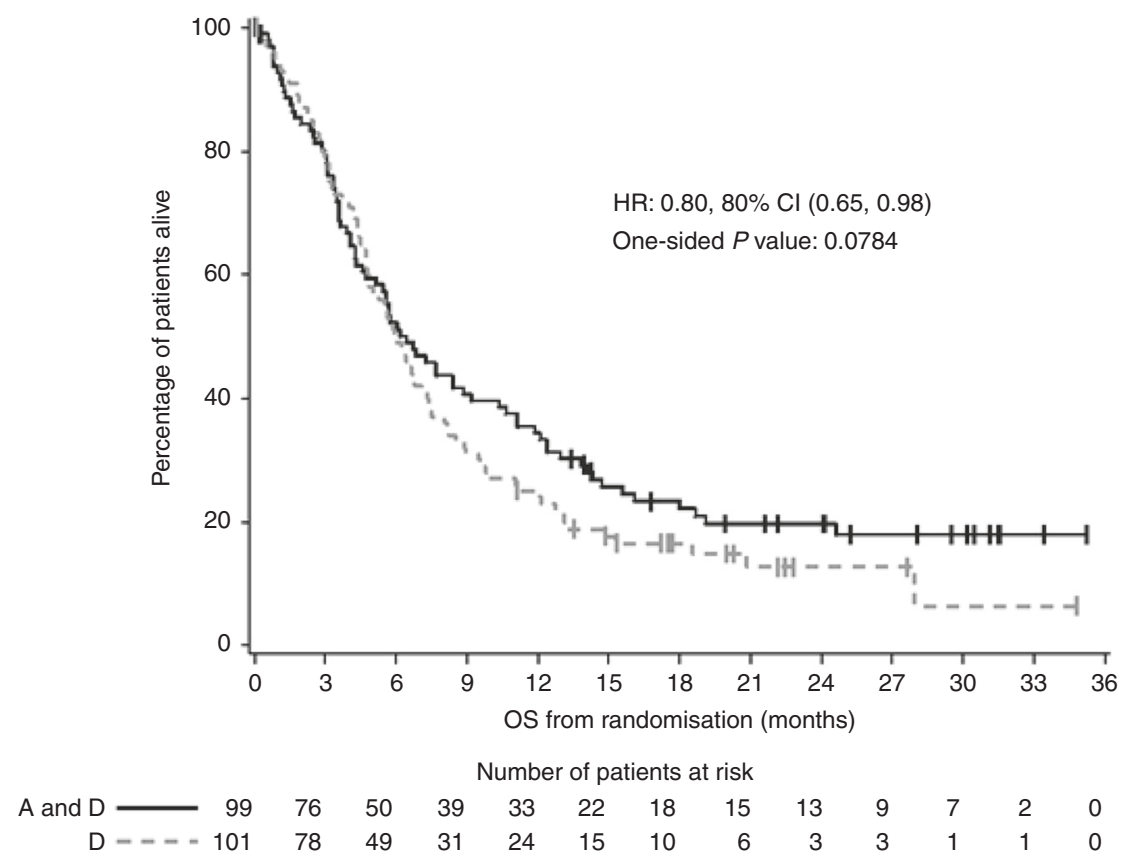

Fig. 2 Kaplan-Meier estimate of overall survival (OS). A apatorsen, D docetaxel, HR hazard ratio, Cl confidence interval

apatorsen, a median of 6 weeks (or doses) of treatment were received. Seven patients went on to receive maintenance apatorsen after stopping docetaxel, with additional weeks of apatorsen reported as: 1, 2, 3, 7, 7, 33, and 61 weeks for these patients.

Primary end point: overall survival

Median follow-up time for all surviving patients was 21.6 months (range, $<1-35.3$ months). At the time of analysis, 163 deaths were reported, with 77 (77.8\%) assigned docetaxel and apatorsen and $86(85.1 \%)$ assigned docetaxel. Patients assigned to docetaxel and apatorsen had a reduction in hazard of death as compared to patients assigned docetaxel alone (HR: $0.80 ; 80 \% \mathrm{Cl}$ : 0.65-0.98, one-sided $P=0.0784$, median OS 6.4 vs 5.9 months). The estimated 12 -month OS was $34.4 \%$ and $25.0 \%$ among patients assigned to docetaxel and apatorsen vs docetaxel alone, respectively (Fig. 2).

Secondary end points and subgroup analysis

Patients assigned to docetaxel and apatorsen had a reduced hazard of disease progression or death as compared to patients assigned to docetaxel alone, although the results were not statistically significant (HR: $0.80,80 \% \mathrm{Cl}$ : $0.64-1.01$, one-sided $P=$ 0.1069 , median PFS 1.8 vs 1.6 months, estimated 12-month PFS $8.7 \%$ vs $3.8 \%)$.

One-hundred and forty-five patients had evaluable disease by RECIST 1.1 criteria. The ORR was $16.1 \%$ (80\% Cl: $11.5-21.9)$ for patients assigned docetaxel and apatorsen vs $10.9 \%(80 \% \mathrm{Cl}$ : 7.1-16.0) for those assigned docetaxel alone (one-sided $P=$ 0.1531). Median duration of response was 6.2 months and 4.4 months in the docetaxel plus apatorsen and docetaxel alone responders, respectively.

Subgroup analysis was performed on patients with Bellmunt prognostic factors 0 vs $1-3$ as well as those with time from prior systemic chemotherapy $<3$ vs $\geq 3$ months. In participants with 0 risk factors, treatment with docetaxel and apatorsen vs docetaxel alone resulted in a median OS 14.3 vs 10.9 months (HR: $0.91,80 \%$ Cl: $0.59-1.42)$, compared to a median OS of 5.6 vs 5.1 months in those with 1-3 risk factors (HR: $0.77,80 \% \mathrm{Cl}: 0.61-0.97$ ). In participants with time from prior systemic chemotherapy $\geq 3$ months, median OS was 8.0 vs 6.7 months for patients assigned docetaxel and apatorsen vs docetaxel alone (HR: 0.89 , $80 \% \mathrm{Cl}: 0.67-1.18)$ and in those with time from prior systemic chemotherapy $<3$ months, the median OS was 5.9 vs 4.7 months (HR: 0.71, 80\% Cl: 0.53-0.96) for patients assigned docetaxel and apatorsen vs docetaxel, respectively.

There was also no significant impact on survival for patients having primary surgery, compared to those that did not (HR: 0.90; $80 \%$ Cl: $0.73-1.12$, one-sided $P=0.2638$ ).

Safety

Of the 200 participants randomised, 189 (93 assigned docetaxel plus apatorsen and 96 assigned docetaxel alone) initiated protocol treatment and were included in the safety population. Overall, 187 (98.9\%) patients experienced an adverse event (AE) of any grade. The most common all-grade AEs were fatigue, anorexia, constipation, diarrhoea, nausea, anaemia, leukopaenia, and neutropaenia (Table 2). Among patients assigned to docetaxel and apatorsen, 77 $(82.8 \%)$ had at least one grade 3-5 AE reported compared with 72 (75.0\%) patients assigned to docetaxel alone. Common grade 3-5 AEs, including neutropaenia, leukopaenia, anaemia, and febrile neutropaenia, were well balanced between both groups. Patients treated with docetaxel and apatorsen had greater incidence of sepsis $(15.1 \%$ vs $8.3 \% ; n=14$ vs 8$)$ and urinary tract infections (14\% vs $7.3 \% ; n=13$ vs 7 ) compared to those treated with docetaxel alone. Thirteen percent of patients discontinued treatment due to unacceptable adverse events $(16.2 \%(n=16)$ assigned docetaxel and apatorsen and 9.9\% $(n=10)$ assigned docetaxel). Five patients in each arm experienced grade $5 \mathrm{AEs}$. In the docetaxel and apatorsen arm, one was possibly drug related to the treatment combination, and one was possibly drug related to apatorsen only. One death in each arm was possibly drug related to docetaxel alone.

Exploratory analyses: serum Hsp27 levels

Of the 200 participants, 161 (80.5\%) had a baseline serum Hsp27 level available (80 in arm A and 81 in arm B). Median Hsp27 level was $5.7 \mathrm{ng} / \mathrm{mL}$. In patients who had a baseline Hsp27 level $<5.7 \mathrm{ng} / \mathrm{mL}(n=79)$, median OS was significantly higher compared to those with a baseline level $\geq 5.7 \mathrm{ng} / \mathrm{mL}(n=82)$ (median 
Table 2. Selected adverse events among the safety population initiating assigned treatment

Treatment assignment

\begin{tabular}{|c|c|c|c|c|c|c|c|c|}
\hline & \multicolumn{4}{|c|}{ Patients given docetaxel and apatorsen $(n=93)$} & \multicolumn{4}{|c|}{ Patients given docetaxel $(n=96)$} \\
\hline & Grades 1-2 & Grade 3 & Grade 4 & Grade 5 & Grades 1-2 & Grade 3 & Grade 4 & Grade 5 \\
\hline Fatigue & $56(60 \%)$ & $7(7 \%)$ & 0 & 0 & $54(56 \%)$ & $12(12 \%)$ & 0 & 0 \\
\hline Diarrhoea & $40(43 \%)$ & $7(7 \%)$ & 0 & 0 & $30(31 \%)$ & $5(5 \%)$ & 0 & 0 \\
\hline Anaemia & $24(26 \%)$ & $16(17 \%)$ & 0 & 0 & $25(26 \%)$ & $10(10 \%)$ & $2(2 \%)$ & 0 \\
\hline Nausea & $39(42 \%)$ & $2(2 \%)$ & 0 & 0 & $31(32 \%)$ & $3(3 \%)$ & 0 & 0 \\
\hline Anorexia & $42(45 \%)$ & 0 & 0 & 0 & $29(30 \%)$ & $1(1 \%)$ & 0 & 0 \\
\hline Neutropaenia & $4(4 \%)$ & $14(15 \%)$ & $19(20 \%)$ & 0 & $4(4 \%)$ & $11(11 \%)$ & $18(19 \%)$ & 0 \\
\hline Constipation & $35(38 \%)$ & $2(2 \%)$ & 0 & 0 & $24(25 \%)$ & $1(1 \%)$ & 0 & 0 \\
\hline Dyspnea & $22(24 \%)$ & $6(6 \%)$ & 0 & 0 & $26(27 \%)$ & $3(3 \%)$ & $1(1 \%)$ & 0 \\
\hline Leukopaenia & $4(4 \%)$ & $18(19 \%)$ & $9(10 \%)$ & 0 & $4(4 \%)$ & $14(14 \%)$ & $5(5 \%)$ & 0 \\
\hline Alopecia & $26(28 \%)$ & 0 & 0 & 0 & $26(27 \%)$ & 0 & 0 & 0 \\
\hline Peripheral neuropathy & 27 (29\%) & $1(1 \%)$ & 0 & 0 & $22(23 \%)$ & 0 & 0 & 0 \\
\hline Creatinine increased & $28(30 \%)$ & $2(2 \%)$ & 0 & 0 & $11(11 \%)$ & $1(1 \%)$ & 0 & 0 \\
\hline Muscle weakness & $14(15 \%)$ & $4(4 \%)$ & 0 & 0 & $14(14 \%)$ & $4(4 \%)$ & 0 & 0 \\
\hline Oral mucositis & $14(15 \%)$ & $1(1 \%)$ & 0 & 0 & $19(20 \%)$ & $2(2 \%)$ & 0 & 0 \\
\hline Vomiting & $16(17 \%)$ & $4(4 \%)$ & 0 & 0 & $13(13 \%)$ & $3(3 \%)$ & 0 & 0 \\
\hline Dysgeusia & $17(18 \%)$ & 0 & 0 & 0 & $17(18 \%)$ & 0 & 0 & 0 \\
\hline Hyponatremia & $16(17 \%)$ & $7(7 \%)$ & 0 & 0 & $7(7 \%)$ & $4(4 \%)$ & 0 & 0 \\
\hline Urinary tract infection & $7(7 \%)$ & $13(14 \%)$ & 0 & 0 & $6(6 \%)$ & $7(7 \%)$ & 0 & 0 \\
\hline Lymphopaenia & $6(6 \%)$ & $11(12 \%)$ & 0 & 0 & $4(4 \%)$ & $6(6 \%)$ & $1(1 \%)$ & 0 \\
\hline Hypertension & $7(7 \%)$ & $4(4 \%)$ & 0 & 0 & $5(5 \%)$ & $8(8 \%)$ & 0 & 0 \\
\hline Thrombocytopaenia & $12(13 \%)$ & 0 & 0 & 0 & $10(10 \%)$ & 0 & 0 & 0 \\
\hline Sepsis & 0 & $3(3 \%)$ & $9(10 \%)$ & $2(2 \%)$ & 0 & $1(1 \%)$ & $7(7 \%)$ & 0 \\
\hline Rash (maculopapular) & $13(14 \%)$ & 0 & 0 & 0 & $8(8 \%)$ & 0 & 0 & 0 \\
\hline Febrile neutropaenia & 0 & $9(10 \%)$ & $1(1 \%)$ & 0 & $2(2 \%)$ & $8(8 \%)$ & 0 & 0 \\
\hline Thromboembolic events & $6(6 \%)$ & $3(3 \%)$ & 0 & 0 & $2(2 \%)$ & $5(5 \%)$ & 0 & 0 \\
\hline $\begin{array}{l}\text { Intracranial } \\
\text { haemorrhage }\end{array}$ & 0 & 0 & 0 & $1(1 \%)$ & 0 & 0 & 0 & $1(1 \%)$ \\
\hline Cardiac arrest & 0 & 0 & 0 & 0 & 0 & 0 & 0 & $1(1 \%)$ \\
\hline Colonic perforation & 0 & 0 & 0 & 0 & 0 & 0 & 0 & $1(1 \%)$ \\
\hline Hepatic failure & 0 & 0 & 0 & 0 & 0 & 0 & 0 & $1(1 \%)$ \\
\hline Death NOS & 0 & 0 & 0 & $1(1 \%)$ & 0 & 0 & 0 & $1(1 \%)$ \\
\hline Multi-organ failure & 0 & 0 & 0 & $1(1 \%)$ & 0 & 0 & 0 & 0 \\
\hline
\end{tabular}

Selected grades 1-2 (in at least $10 \%$ of patients) and grades 3,4 , and 5 adverse events. NOS not otherwise specified

OS 9.4 vs. 4.7 months, HR: $0.51,80 \% \mathrm{Cl}: 0.41-0.65$, one-sided $P=$ 0.0001 , estimated 12 -month OS $43.6 \%$ vs $15.2 \%$ ) (Fig. 3 ). Treatment with docetaxel and apatorsen improved survival in both groups of patients with either baseline Hsp27 level $<5.7 \mathrm{ng} / \mathrm{mL}$ (HR: $0.71,80 \% \mathrm{Cl}: 0.50-1.00$ ) or $\geq 5.7 \mathrm{ng} / \mathrm{mL}$ (HR: $0.67,80 \% \mathrm{Cl}$ : 0.48-0.92; two-sided $P=0.87$ for interaction) compared to docetaxel alone.

A landmark analysis was performed after cycle 2 to evaluate the change in Hsp27 level from baseline. Eighty patients (40 in each treatment group) had measurements available at both time points and median percentage change in serum $\mathrm{Hsp} 27$ from baseline to end of cycle 2 was an increase of $20.5 \%$ (range, -76.8 to $+677.8 \%)$. In patients with a decrease or $\leq 20.5 \%$ increase of Hsp27, treatment with docetaxel and apatorsen significantly improved overall survival vs docetaxel alone (HR: $0.29,80 \% \mathrm{Cl}$ : $0.18-0.48$, median OS 12.2 vs 5.1 months) compared to those who had $>20.5 \%$ increase in Hsp27 levels (HR: $0.77,80 \% \mathrm{Cl}$ : 0.46-1.30, median OS 7.9 vs 6.8 months; two-sided $P=0.0727$ for interaction).

\section{DISCUSSION}

The addition of apatorsen to docetaxel chemotherapy met its predefined end point resulting in improved overall survival compared to docetaxel alone in patients with metastatic urothelial carcinoma who are relapsed or refractory after a platinumcontaining regimen. Although the improvement in median overall survival was 2 weeks, the hazard ratio and Kaplan-Meier curves show the overall benefit for the addition of apatorsen. However, these data are hypothesis generating and confirmatory trials are warranted to further study this targeted agent in patients with metastatic urothelial carcinoma.

Currently in the United States, single-agent immune checkpoint blockade with anti-PD-1/PD-L1 antibodies is the standard of care for patients following progression on platinum-based chemotherapy, ${ }^{22-24}$ and vinflunine is a cytotoxic approved for this indication in the European Union. ${ }^{25}$ While the development of immune checkpoint blockade has represented a breakthrough for patients with advanced urothelial carcinoma, the vast majority of patients fail to respond to single-agent PD-1 or PD-L1 inhibition with 


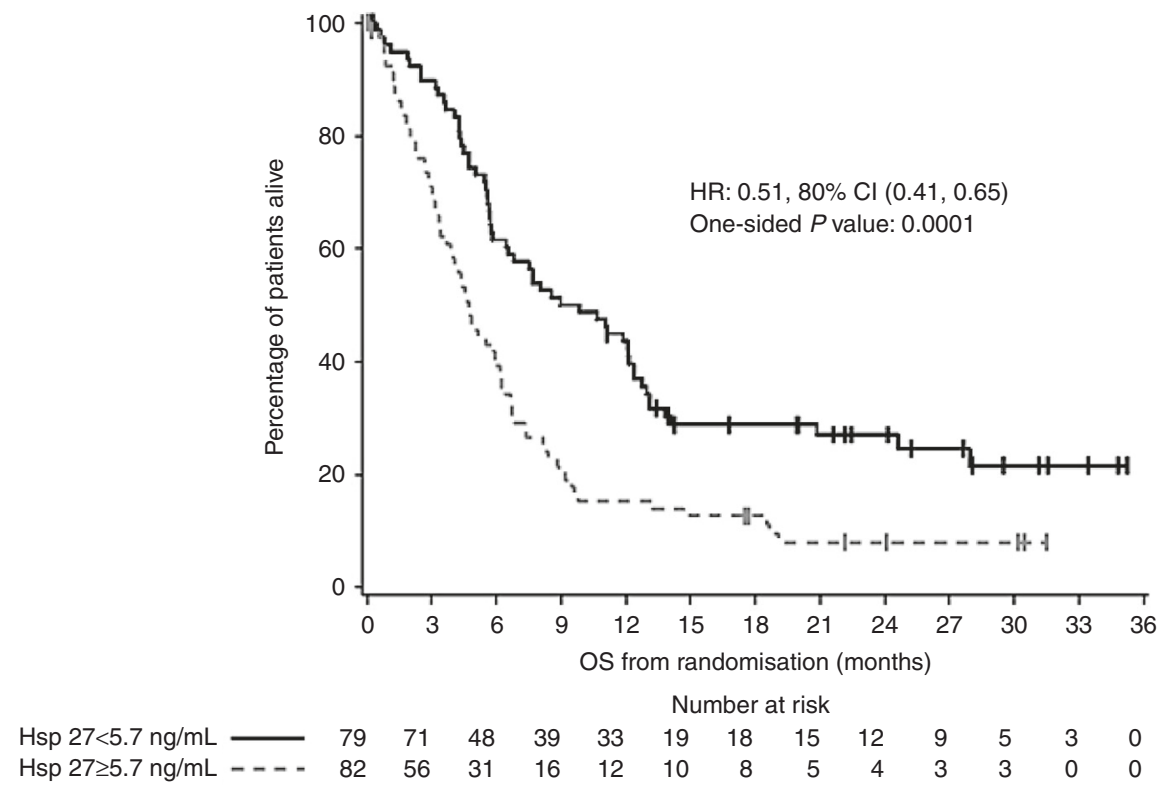

Fig. 3 Kaplan-Meier estimate of overall survival (OS) according to baseline serum Hsp27 levels. One-hundred and sixty-one patients (80.5\%) had baseline Hsp27 levels available. HR hazard ratio, $\mathrm{Cl}$ confidence interval

response rates of $15-25 \% .{ }^{22-24,26,27}$ For these patients, there are no proven life-prolonging treatments, and the outlook remains quite bleak. Furthermore, efficacy results from phase II trials of many of these agents, which have led to accelerated FDA approval status, remain to be rigorously verified in the phase III setting and, in some cases, have not been confirmed in the subsequent phase trial. ${ }^{28}$ The phase III RANGE study, which evaluated ramucirumab plus docetaxel in platinum-refractory metastatic urothelial carcinoma, showed a modest PFS improvement of only 1.3 months compared to docetaxel plus placebo. ${ }^{29}$ Due to its gate-keeping statistical design, the study does not allow for formal testing of response rate unless OS benefit reaches HR 0.75 (currently immature). There were limited number of patients receiving immune checkpoint blockade prior and subgroup analysis did not show any benefit in patients with visceral metastasis. Therefore, novel agents that target chemotherapy-resistant urothelial carcinoma are still desperately needed.

Preclinical and smaller clinical data have shed light on the rationale for activity and benefit from ASO therapy targeting Hsp27. Hsp27 is a stress-activated, ATP-independent, cytoprotective chaperone that is upregulated in cancer and is associated with treatment resistance. Inhibition of Hsp27 expression in vitro and in vivo leads to increased sensitivity to cytotoxic chemotherapies. ${ }^{14,15}$ In addition, suppression of Hsp27 may lead to long-term dormancy in vivo in the absence of chemotherapy though inhibition of angiogenesis. ${ }^{30}$ Collectively, these findings may provide rationale for outcomes observed on this trial: while the median difference in overall survival was small, the hazard ratio suggests a $20 \%$ reduction in the risk of death over the course of the study associated with the combination treatment arm.

The biology of Hsp27 and its targeting by ASO has been shown to have challenges. Through the interplay of TNF- $a$ and IL-10, Hsp27 is known to have anti-inflammatory effects. ${ }^{31}$ Further, while second-generation ASOs have modifications to allow greater nuclease resistance and increased binding affinities than their predecessors, potential toxicities include hybridisation-dependent toxicities-due to on- or off-target pharmacology-and hybridisation-independent toxicities due to nonantisense effects. ${ }^{32}$ Inhibition of Hsp27 by ASOs, therefore, may result in tumour suppression at the expense of increased inflammation. Indeed, in our study we noted a slightly higher incidence of sepsis
(15.1\% vs $8.3 \% ; n=14$ vs 8 ) and urinary tract infections ( $14 \%$ vs $7.3 \% ; n=13$ vs 7 ) in those treated with combination therapy. These safety signals are generally consistent with those seen in the Borealis-1 study, which evaluated platinum-based chemotherapy with or without apatorsen (600 or $1000 \mathrm{mg}$ ) vs chemotherapy plus placebo in the first-line setting. ${ }^{20}$ Toxicity was noted to be higher in the $1000 \mathrm{mg}$ apatorsen arm compared to the $600 \mathrm{mg}$ arm in that study, although the primary end point of improved OS was not met with either apatorsen dose compared to chemotherapy alone. In the phase III AFFINITY study of men with metastatic castration-resistant prostate cancer who progressed after docetaxel, custirsen, an ASO to clusterin, administered with chemotherapy showed no OS benefit vs chemotherapy alone. ${ }^{33}$ Our study, however, met its predefined end point and the future prospect of ASOs in urothelial carcinoma may depend on better patient selection, for example, with accurate biomarkers.

In our subgroup analysis, patients with lower baseline serum Hsp27 levels $(<5.7 \mathrm{ng} / \mathrm{mL})$ appeared to have better overall survival than those with higher baseline levels $(\geq 5.7 \mathrm{ng} / \mathrm{mL})$, irrespective of treatment. Therapy with apatorsen and docetaxel appeared to benefit both those with lower or higher Hsp27 levels, suggesting that baseline serum Hsp27 levels may act as a potential prognostic, but not predictive, biomarker in these patients. Furthermore, combination treatment with apatorsen and docetaxel appeared to benefit those with either a decline or limited increase of Hsp27 level $\leq 20.5 \%$ (HR: $0.29,80 \%$ Cl: $0.18-0.48$ ) more so than those with an increase of $>20.5 \%$ (HR: $0.77,80 \% \mathrm{Cl}$ : $0.46-1.30$ ) when comparing baseline and post cycle 2 levels. This finding may suggest that dynamic changes of Hsp27 levels in patients could serve as an indicator predicting benefit to combination treatment. Ultimately, these findings are simply hypothesis generating and may be informative for future trial designs.

These results should be interpreted in the context of study design. This was a randomised, controlled, comparative trial with one-sided 0.10 alpha-level test reflecting the objective to determine if the combination provided survival benefit relative to docetaxel alone. The rationale for this statistical design was that the addition of apatorsen to docetaxel was not felt to potentially yield a negative effect compared to docetaxel alone; however, this does allow for a higher false-positive rate and there is a potential 
for lead time bias with the apatorsen run-in. In the context of recent phase III results for immunotherapy agents in this space, our findings reinforce the importance of OS as a significant end point in well-designed later phase studies when evaluating potential practice-impacting treatments. ${ }^{28}$ To be eligible for our trial, patients must have received at least one prior platinumbased chemotherapy regimen, and no patient may have received more than two regimens for metastatic disease. While the current landscape is evolving for patients who are platinum-ineligible, and there are new options in the platinum-refractory setting (e.g., immunotherapy), this was not part of established treatment paradigm at the time of our study design. Therefore, the efficacy of apatorsen in patients having received immunotherapy is not currently assessed and granular data on subsequent lines of therapy are not available in this analysis. Furthermore, we only analysed serum Hsp27 levels at baseline and after cycle 2 as a potential marker of response to treatment. Evaluating the expression of Hsp27 measured by immunohistochemistry (IHC) in tumour tissue, as well as the effect of therapy on peripheral circulating tumour cells (CTCs), would be meaningful additional exploratory end points and, while not available in this current report, are planned future analyses.

In conclusion, the addition of apatorsen to docetaxel chemotherapy met its predefined survival end point in patients with refractory metastatic urothelial carcinoma in this phase II trial. These data are hypothesis generating and would require further study before informing clinical practice for this targeted therapy in metastatic urothelial carcinoma.

\section{ACKNOWLEDGEMENTS}

The authors acknowledge the following investigators for their participation in this study: Madelaine Sgroi (IU Health Central Indiana Cancer Centers); Ralph Hauke (Nebraska Methodist Hospital); Arif Hussain (University of Maryland); John Burfeind (Froedtert \& The Medical College of Wisconsin); Alexander Starodub (Goshen Center for Cancer Care); Alexandra Drakaki (University of California Los Angeles); Sergey Devitskiy (Dartmouth-Hitchcock Medical Center); Harry Drabkin (Medical University of South Carolina); William Adler (Memorial Medical Cancer Center, Las Cruces); David Quinn (University of Southern California); William Fisher (IU Health Ball Memorial Hospital Cancer Center). The authors also acknowledge Robert Dreicer, currently at University of Virginia, and Roberto Pili, currently at Indiana University, for their contributions.

\section{AUTHOR CONTRIBUTIONS}

J.E.R., N.M.H., T.K.C., M.M.R and L.W. designed this trial. J.E.R., N.M.H., A.A., S.G., J.P., R. A., A.B., J.H.-C., P.G., R.L., E.G., C.H., G.S., C.A., M.S., J.B., S.P., and T.K.C. recruited patients. M.M.R. provided statistical leadership, L.W. implemented the statistical analysis, and T. B. oversaw data management. A.A.L., J.E.R., N.M.H., M.M.R., L.W., and T.K.C. drafted the manuscript. C.J. and K.A. are OncoGenex employees. All authors contributed to the interpretation of data, reviewing the manuscript, and gave final approval to submit for publication.

\section{ADDITIONAL INFORMATION}

Conflict of interest: J.E.R. reports personal fees from OncoGenex during the conduct of the study; personal fees from Merck, BMS, Eli Lilly, AstraZeneca, EMD Serono, Inovio, Agensys, Genentech/Roche, Seattle Genetics, Sanofi, outside the submitted work. N.M.H. reports research support to the institution from Novartis, OncoGenex, Mirati, Merck, Genentech, Bristol-Myers Squibb, Heat Biologics, Acerta, AstraZeneca, and Prinicipia Biopharma; consulting support from OncoGenex, AstraZeneca, Merck, Bristol-Myers Squibb, Genentech, Inovio, Principia Biopharma, Champions Oncology, Health Advances, Taris Biomedical, and Seattle Genetics. M.M.R. reports grants from Hoosier Cancer Research Network during the conduct of the study. S.G. reports grants from BMS, Novartis, Bayer, Agensys, Merck, Pfizer, and Celldex during the conduct of the study; personal fees from AstraZeneca, Bristol-Myers Squibb, Bayer, Exelixis, Novartis, and Pfizer, outside the submitted work. J.P. reports institutional research funding from OncoGenex and Hoosier Cancer Research Network. A.B. reports research support to the institution from Merck, Genentech, and AstraZeneca; consulting support from AstraZeneca, Merck, Genentech, Pfizer/EMD Serono, and Cerulian Pharma. J.H.-C. reports grants and personal fees from Genentech; non- financial support from Genentech; personal fees from Clovis Oncology, Novartis, Merck, and Foundation Medicine, outside the submitted work. P.G. reports consulting with Genentech, Bayer, Dendreon, Bristol-Myers Squibb, Merck, Exelixis, AstraZeneca, Clovis Oncology, and EMD Serono; also participated in unbranded educational program with Genentech and Bristol-Myers Squibb. G.S. reports grants from Boehringer-Ingelheim, Bayer, Onyx-Amgen, and Merck; consultancy fees from Pfizer, Genentech, Novartis, Argos, Merck, Sanofi, Agensys, Clinical Care Options, Astrazeneca, Uptodate, Biotheranostics, Exelixis, Bristol-Myers Squibb, Janssen, Amgen, and Eisai; personal fees from NCCN (National Comprehensive Cancer Network). T.B. is a Hoosier Cancer Research Network employee. C.J. and K.A. are OncoGenex employees. J.B. reports research support from Novartis and Sanofi; consulting support from OncoGenex, AstraZeneca, Merck, Bristol-Myers Squibb, Genentech, Inovio, Champions Oncology, Seattle Genetics, and Pierre Fabre. T.K.C. reports institutional research funding from Pfizer, Exelixis, BMS, and Novartis; advisory roles at Pfizer, Novartis, Genentech, Merck, BMS, and Bayer. The remaining authors $L$. W., A.A., R.A., R.L., E.A.G., C.H., C.A., M.N.S., A.A.L., and S.P. declare no competing interests.

Availability of data and material: All data and materials are available upon request.

Ethical approval and consent to participate: The study was approved by the institutional review boards and/or ethics committee at every enrolling center and was conducted in accordance with Good Clinical Practice Guidelines and the Declaration of Helsinki. The study was reviewed every 6 months by the Data Safety Monitoring Board of the Dana-Farber/Harvard Cancer Center.

Funding: This study was investigator-sponsored with support from OncoGenex Pharmaceuticals. This work was supported in part by National Cancer Institute Cancer Center Support grant P30 CA008748. The study was designed by the three principal investigators (J.E.R., N.M.H., and T.K.C.). The study was conducted among academic and community sites within the Hoosier Cancer Research Network (HCRN). None of the funders had any role in the data analysis, interpretation of results, or a role in writing the article. The principal investigators and statisticians (L.W. and M.M.R.) had access to raw data. The principal investigators had the final responsibility for the decision to submit for publication.

Note: This work is published under the standard license to publish agreement. After 12 months the work will become freely available and the license terms will switch to a Creative Commons Attribution 4.0 International licence (CC BY 4.0).

\section{REFERENCES}

1. Ischia, J. \& So, A. I. The role of heat shock proteins in bladder cancer. Nat. Rev. Urol. 10, 386-395 (2013).

2. Miyata, Y. Hsp90 inhibitor geldanamycin and its derivatives as novel cancer chemotherapeutic agents. Curr. Pharm. Des. 11, 1131-1138 (2005).

3. Whitesell, L. et al. Geldanamycin-stimulated destabilization of mutated p53 is mediated by the proteasome in vivo. Oncogene 14, 2809-2816 (1997).

4. An, W. G., Schulte, T. W. \& Neckers, L. M. The heat shock protein 90 antagonist geldanamycin alters chaperone association with $\mathrm{p} 210 \mathrm{bcr}$-abl and v-src proteins before their degradation by the proteasome. Cell Growth Differ. 11, 355-360 (2000).

5. Workman, P. Overview: translating Hsp90 biology into Hsp90 drugs. Curr. Cancer Drug Targets 3, 297-300 (2003).

6. Bagatell, R. \& Whitesell, L. Altered Hsp90 function in cancer: a unique therapeutic opportunity. Mol. Cancer Ther. 3, 1021-1030 (2004).

7. Whitesell, L. \& Lindquist, S. L. HSP90 and the chaperoning of cancer. Nat. Rev. Cancer 5, 761-772 (2005).

8. Somji, S., Sens, D. A., Garrett, S. H., Sens, M. A. \& Todd, J. H. Heat shock protein 27 expression in human proximal tubule cells exposed to lethal and sublethal concentrations of CdCl2. Environ. Health Persspect. 107, 545-552 (1999).

9. Storm, F. K., Mahvi, D. M. \& Gilchrist, K. W. Hsp-27 has no diagnostic or prognostic significance in prostate or bladder cancers. Urology 42, 379-382 (1993).

10. Lebret, T. et al. Heat shock proteins HSP27, HSP60, HSP70, and HSP90: expression in bladder carcinoma. Cancer 98, 970-977 (2003).

11. Kassem, HSh, Sangar, V., Cowan, R., Clarke, N. \& Margison, G. P. A potential role of heat shock proteins and nicotinamide $\mathrm{N}$-methyl transferase in predicting response to radiation in bladder cancer. Int. J. Cancer 101, 454-460 (2002).

12. Gleave, M., Nelson, C. \& Chi, K. Antisense targets to enhance hormone and cytotoxic therapies in advanced prostate cancer. Curr. Drug Targets 4, 209-221 (2003).

13. Jansen, B. \& Zangemeister-Wittke, U. Antisense therapy for cancer-the time of truth. Lancet Oncol. 3, 672-683 (2002). 
14. Kamada, M. et al. Hsp27 knockdown using nucleotide-based therapies inhibit tumour growth and enhance chemotherapy in human bladder cancer cells. Mol. Cancer Ther. 6, 299-308 (2007).

15. Hadaschik, B. A. et al. Intravesically administered antisense oligonucleotides targeting heat-shock protein-27 inhibit the growth of non-muscle-invasive bladder cancer. BJU Int. 102, 610-616 (2008).

16. So, A. I., Black, P. C., Chi, K. N., Hurtado-Col, A. \& Gleave, M. E. A phase I trial of intravesical antisense oligonucleotide targeting heat shock protein 27 (OGX-427) for the treatment of non-muscle-invasive bladder cancer. J. Clin. Oncol. 30, 286 (2012).

17. Chi, K. N. et al. A phase I dose-escalation study of apatorsen (OGX-427), an antisense inhibitor targeting heat shock protein 27 ( $\mathrm{Hsp} 27$ ), in patients with castration-resistant prostate cancer and other advanced cancers. Ann. Oncol. 27, 1116-1122 (2016).

18. Bellmunt, J. et al. Prognostic factors in patients with advanced transitional cell carcinoma of the urothelial tract experiencing treatment failure with platinumcontaining regimens. J. Clin. Oncol. 28, 1850-1855 (2010).

19. Sonpavde, G. et al. Time from prior chemotherapy enhances prognostic risk grouping in the second-line setting of advanced urothelial carcinoma: a retrospective analysis of pooled, prospective phase 2 trials. Eur. Urol. 63, 717-723 (2013).

20. Bellmunt, J. et al. Borealis-1: a randomised, first-line, placebo-controlled, phase II study evaluating apatorsen and chemotherapy for patients with advanced urothelial cancer. Ann. Oncol. 28, 2481-2488 (2017).

21. Choueiri, T. K. et al. Double-blind, randomised trial of docetaxel plus vandetanib versus docetaxel plus placebo in platinum-pretreated metastatic urothelial cancer. J. Clin. Oncol. 30, 507-512 (2012).

22. Rosenberg, J. E. et al. Atezolizumab in patients with locally advanced and metastatic urothelial carcinoma who have progressed following treatment with platinum-based chemotherapy: a single-arm, multicentre, phase 2 trial. Lancet 387, 1909-1920 (2016).

23. Sharma, P. et al. Nivolumab in metastatic urothelial carcinoma after platinum therapy (CheckMate 275): a multicentre, single-arm, phase 2 trial. Lancet Oncol. 18, 312-322 (2017)
24. Bellmunt, J. et al. Pembrolizumab as second-line therapy for advanced urothelial carcinoma. N. Engl. J. Med. 376, 1015-1026 (2017).

25. Bellmunt, J. et al. Phase III trial of vinflunine plus best supportive care compared with best supportive care alone after a platinum-containing regimen in patients with advanced transitional cell carcinoma of the urothelial tract. J. Clin. Oncol. 27, 4454-4461 (2009).

26. Powles, T. et al. Efficacy and safety of durvalumab in locally advanced or metastatic urothelial carcinoma: updated results from a phase 1/2 open-label study. JAMA Oncol. 3, e172411 (2017).

27. Patel, M. R. et al. Avelumab in metastatic urothelial carcinoma after platinum failure (JAVELIN solid tumour): pooled results from two expansion cohorts of an open-label phase 1 trial. Lancet Oncol. 19, 51-64 (2018).

28. Powles, T. et al. Atezolizumab versus chemotherapy in patients with platinumtreated locally advanced or metastatic urothelial carcinoma (IMvigor 211): a multicenter, open-label, phase 3 randomised controlled trial. Lancet 391 , 748-757 (2018).

29. Petrylak, D. P. et al. Ramucirumab plus docetaxel versus placebo plus docetaxel in patients with locally advanced or metastatic urothelial carcinoma after platinumbased therapy (RANGE): a randomised, double-blind, phase 3 trial. Lancet $\mathbf{3 9 0}$, 2266-2277 (2017).

30. Straume, O. et al. Suppression of heat shock protein 27 induces long-term dormancy in human breast cancer. Proc. Natl Acad. Sci. USA 109, 8699-8704 (2012)

31. De, A. K., Kodys, K. M., Yeh, B. S. \& Miller-Graziano, C. Exaggerated human monocyte IL-10 concomitant to minimal TNF-alpha induction by heat-shock protein 27 (Hsp27) suggests Hsp27 is primarily an antiinflammatory stimulus. J. Immunol. 165, 3951-3958 (2000).

32. Frazier, K. S. Antisense oligonucleotide therapies: the promise and the challenges from a toxicologic pathologist's perspective. Toxicol. Pathol. 43, 78-89 (2015).

33. Beer, T. M. et al. Custirsen (OGX-011) combined with cabazitaxel and prednisone versus cabazitaxel and prednisone alone in patients with metastatic castrationresistant prostate cancer previously treated with docetaxel (AFFINITY): a randomised, open-label, international, phase 3 trial. Lancet Oncol. 18, 1532-1542 (2017). 